\title{
Mouse Period1 (mPER1) Acts as a Circadian Adaptor to Entrain the Oscillator to Environmental Light/Dark Cycles by Regulating mPER2 Protein
}

\author{
Satoru Masubuchi, ${ }^{1}$ Noritoshi Kataoka, ${ }^{1}$ Paolo Sassone-Corsi, ${ }^{2}$ and Hitoshi Okamura ${ }^{1}$ \\ ${ }^{1}$ Division of Molecular Brain Science, Department of Brain Sciences, Kobe University Graduate School of Medicine, Chuo-ku, Kobe 650-0017, Japan, and \\ ${ }^{2}$ Institut de Génétique et de Biologie Moléculaire et Cellulaire, Centre National de la Recherche Scientifique, Institut National de la Santé et de la Recherche \\ Médicale, Université Louis Pasteur, 67404 Illkirch-Strasbourg, France
}

\begin{abstract}
Mouse period1 (mPer1) and mPer2 are mammalian homologs of the Drosophila clock gene period that show robust oscillation in the suprachiasmatic nucleus, the mammalian master clock, and have been implicated as essential components of the core clock mechanism. Gene-targeting studies have demonstrated that $m$ Per 2 plays a dominant function in behavioral rhythm generation, although the role of $m$ Per 1 has not been fully clarified. Here, we report that prolongation of the lighting period $(4-16 \mathrm{~h})$ induces a larger-delay phase shift of the behavioral rhythm in mPerl-deficient $\left(\mathrm{mPer}^{-1-}\right)$ mice. During the light-elongation task, mPER2 protein decay in $m P e r 1^{-1-}$ mice is slower $(\sim 4 \mathrm{~h})$ than in wild-type mice, which thereby causes larger behavioral phase delay. $m P e r 1^{-1-}$ mice could not adapt to environmental light/dark cycles in long complete photoperiods with dim light or in long skeleton photoperiods. These photoperiodic conditions mimic natural environmental changes present at high latitudes, indicating that mPerl could operate in the adaptation of the circadian clock of nocturnal mice to large seasonal changes of environmental light/dark cycles.
\end{abstract}

Key words: hypothalamus; suprachiasmatic; circadian; behavior; mPer1; mPer2

\section{Introduction}

Mouse period1 (mPer1) and mPer2 are mammalian homologs of the Drosophila clock gene period (for review, see Dunlap, 1999) that show robust oscillation in the suprachiasmatic nucleus (SCN), the mammalian master clock (Klein et al., 1991), and have been implicated as essential components of the core clock mechanism. Gene-targeting studies have demonstrated that mPer2 knock-out mice are arrhythmic in constant darkness (DD) (Zheng et al., 1999; Bae et al., 2001), whereas mPer1 knock-out $\left(m P e r 1^{-/-}\right)$mice elicit a persistent rhythmicity with a slightly shorter period length (Bae et al., 2001; Cermakian et al., 2001; Zheng et al., 2001). Although it is demonstrated that mPer1 has a crucial role for rhythm generation in peripheral clocks (Cermakian et al., 2001; Pando et al., 2002), its function in the central oscillator remains unclear.

Phase shifting by light is an essential feature of circadian rhythms (Daan and Pittendrigh, 1976a). The phase-shift profiles

Received Nov. 21, 2004; revised March 31, 2005; accepted March 31, 2005.

This work was supported by the Scientific Research Fund from the Ministry of Health, Labor and Welfare of Japan (H.O.), by SRF (H.O.), by grants from the Special Coordination Funds on Priority Areas of the Ministry of Education, Culture, Sports, Science and Technology of Japan (H.O.), by the Centre National de la Recherche Scientifique, Institut National de la Santé et de la Recherche Médicale, Centre Hospitalier Universitaire Régional, Fondation de la Recherche Médicale, Université Louis Pasteur, Human Frontier Science Program (RG-240), and Ligue contre le Cancer (P.S.-C.), and by a grant-in-aid for Encouragement of Young Scientists from the Japan Society for the Promotion of Science (S.M.). We thank W. Schwartz for discussions and H. Isejima for assistance.

Correspondence should be addressed to Dr. Hitoshi Okamura, Division of Molecular Brain Science, Department of Brain Sciences, Kobe University Graduate School of Medicine, 7-5-1 Kusunoki-cho, Chuo-ku, Kobe 650-0017, Japan. E-mail: okamurah@kobe-u.ac.jp.

D0I:10.1523/JNEUROSCI.4761-04.2005

Copyright $\odot 2005$ Society for Neuroscience $\quad$ 0270-6474/05/254719-06\$15.00/0 arising from short exposure to light do not show a difference between $m P e r 1^{-/-}$and wild-type $\left(m P e r 1^{+/+}\right)$mice (Cermakian et al., 2001; Spoelstra et al., 2004), although a decrease in phase advances was noted just after the move to DD (Albrecht et al., 2001; Spoelstra et al., 2004). Because it is considered that the larger the duration of light exposure, the greater the phase shift that occurs (Daan and Pittendrigh, 1976b), in the present study, we adopted long light exposure as a task for revealing the role of $m P e r 1$ to detect the difference between $m P e r 1^{-1-}$ and $m P e r 1^{+/+}$ mice. Here, we found that the magnitude of phase delays arising from long light in $\mathrm{mPer}^{-1-}$ mice was larger than that of wildtype mice, accompanying the delay of mPER 2 protein decay without effecting a change at the mRNA level in the initial phase during the light exposure. The altered core clock machinery in these mice was evident in long complete photoperiods with dim light or in long skeleton photoperiods in which animals show free-running rhythms not adapting to environmental cycles.

\section{Materials and Methods}

Animals and behavioral rhythm monitoring. mPer ${ }^{+/+}$, heterozygous mutant $\left(\mathrm{mPer}^{+/-}\right)$, and $m$ Per $1^{-1-}$ mice (Cermakian et al., 2001) were bred and housed under light/dark (LD) $12 \mathrm{~h}$ cycles (fluorescent light, 200-300 lux). Locomotor activity was detected by passive (pyroelectric) infrared sensors (FA-05 F5B; Omron, Kyoto, Japan) (Shigeyoshi et al., 1997). Data were monitored and analyzed as described previously (Masubuchi et al., 2000) by Chronobiology kit (Stanford Software Systems, Stanford, CA). The experimental protocol of the current research was approved by the Committee for Animal Research at Kobe University.

In situ hybridization. In situ hybridization histochemistry using the free-floating sections was performed according to the method detailed 
previously (Shigeyoshi et al., 1997). We used ${ }^{33} \mathrm{P}$-radiolabeled cRNA probes for $m P e r 2(\mathrm{Ta}-$ kumi et al., 1998) and albumin gene D-sitebinding protein $(d b p)$ (Yamaguchi et al., 2000) for the in situ hybridization studies. The peak value of $m P e r 1^{+/+}$mice was adjusted to 100 , and relative RNA abundance was used.

Immunocytochemistry. Immunocytochemistry was performed with the avidin-biotin-peroxidase method applied to free-floating sections (Ban et al., 1997). Serial frontal sections (30 $\mu \mathrm{m}$ thick) from the rostral end to the caudal end of the SCN were incubated for immunostaining with $1 \mu \mathrm{g} / \mathrm{ml}$ anti-mPER2 (affinitypurified rabbit antisera; Alpha Diagnostic International, San Antonio, TX) (Matsuo et al., 2003), which was finally visualized brown with 3,3'-diaminobenzidine tetrahydrochloride. We counted the mean number of immunoreactive cells in three sections in the middle portion of the SCN. For each point, we used four animals.

Statistical analysis. The $24 \mathrm{~h}$ mRNA and protein variations were statistically tested by oneway ANOVA. The effects of genotypes on the behavioral rhythm onsets were tested by twoway ANOVA. A post hoc Bonferroni/Dunn test was used for the comparison between the values of Perl ${ }^{+/+}, \operatorname{Perl}^{+/-}$, and Per1 ${ }^{-/-}$groups at the same time points. The effects of genotypes on the entrainment daily lighting tasks (complete and skeleton photoperiod) were tested by Fisher's exact probability test.

\section{Results}

Extended light exposure induces larger phase delay of behavioral rhythm in mPer $1^{-/-}$mice mPer $1^{+/+}$, mPer $1^{+/-}$, and mPer $1^{-/-}$ mice (Cermakian et al., 2001) entrained to an LD cycle (12 h; fluorescent light, 200300 lux) were exposed to $0,4,8,12$, and $16 \mathrm{~h}$ of light prolongation (LP) (task LP00, LP04, LP08, LP12, and LP16, respectively) from lights off [Zeitgeber time 12 (ZT12)] at the last day of LD. Afterward, mice were kept in DD. We defined the lights-off points of each task as L00 (corresponding to ZT12), L04 (ZT16), L08 (ZT20), L12 (ZT24), and L16 (ZT28, ZT4 of the next cycle), respectively. The phase-delay effect of the prolongation of light of the last day on the behavioral rhythm was evaluated using extrapolated eye-fitted lines made by $>10 \mathrm{~d}$ of activity onsets. Only the switch from LD to DD does not shift the onset of the extrapolated rhythm in both in $\mathrm{mPer}^{+/+}$and $m P e r 1^{-1-}$ mice, when there was no light prolongation (LP00) (Fig. $1 A$, left). The onset of the behavioral rhythm was delayed in proportion to the increase of the duration of the light exposure (LP04, LP08, LP12) in all three groups $\left(m P e r 1^{+/+}, m P e r 1^{+/-}\right.$, and $m P e r 1^{-I-}$ mice; one-way ANOVA; $p<0.0001$ ) (Fig. $1 B$ ). The phase-delay effect peaked at $12 \mathrm{~h}$ (LP12) and then decreased at $16 \mathrm{~h}$ (LP16). Interestingly, depending on the genotype, the elongation of light exposure differentially affects the magnitude of the phase delay. As shown in Figure $1 A$, when mice were exposed to light prolongation for $12 \mathrm{~h}$ (LP12) for $1 \mathrm{~d}$, the onset of activity rhythm in $m P e r 1^{-1-}$ mice was shifted by $\sim 12 \mathrm{~h}$, strikingly different from $m P e r 1^{+/+}$mice $(\sim 5 \mathrm{~h})$. The effects of elongation of light exposure on the phase delay were significantly



\section{L00L04L08L12L16}

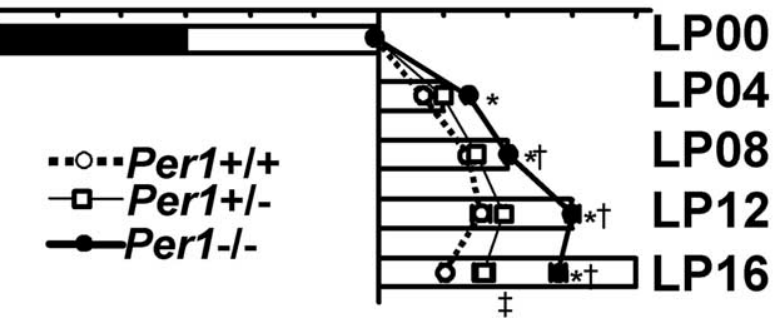

Figure 1. A, Double-plotted locomotor activity rhythms of $m P e r 1^{+/+}$mice $(+/+)$and $m P e r 1^{-1-}$ mice $(-/-)$. Mice were (LP12). Locomotor activities were expressed in the histogram. Periods of darkness are indicated by gray backgrounds. $\boldsymbol{B}$, The effect of light tasks (LP00, LP04, LP08, LP12, LP16) to activity rhythms in $m P e r 1^{+/+}, \mathrm{mPer}^{+/-}$, and $\mathrm{mPer} 1^{-/-}$mice. Extrapolated (me first day are indicated by open circles (mPer $\left.{ }^{+/+}\right)$, open squares (mPer1), and filled circles $\left(\right.$ PPer $\left.^{-1-}\right)$ (mean \pm SEM). For each point, we used five or six animals. The filled bar is the dark phase of LD cycle; open bars are ing phase of $L D$ cycle and light exposure time of each tasks. The values indicated by asterisks and daggers are statistically significant. ${ }^{*} p<0.0001, m$ Per $1^{+/+}$versus $m P e r 1^{-1-} ;{ }^{\dagger} p<0.0001, m P e r 1^{+/+}$versus mPer $1^{+1-} ;{ }^{\ddagger} p<0.0001, m P e r 1^{-1-}$ versus $m P e r 1^{+1-}$ (Bonferroni/Dunn).

different between genotypes (two-way ANOVA; $m P e r 1^{-1-}$ vs $m P e r 1^{+/+}, p<0.0001 ; m P e r 1^{-/-}$vs $m P e r 1^{+/-}, p<0.0001$; $m P e r 1^{+/+}$vs $\left.m P e r 1^{+/-}, p<0.01\right)$ : the behavioral phase delay is much larger in $m P e r 1^{-1-}$ than $m P e r 1^{+/+}(p<0.0001$ in LP04, LP08, LP12, and LP16 tasks) and than $m P e r 1^{+/-}(p<0.0001$ in LP08, LP12, and LP16 tasks) and slightly larger in $\mathrm{mPer}^{+/-}$than $m P e r 1^{+/+}(p<0.0001$ in LP16 task) (Fig. $1 B)$. The prolongation of light exposure $(4-16 \mathrm{~h})$ produces larger phase delay of behavioral rhythm in $m$ Perl $^{-1-}$ mice.

Extended light exposure does not yield differences in mPer2 gene expression in the $\mathrm{SCN}$ of mPer $^{-/-}$mice

Because the SCN is the master clock that times rhythmicity at the systemic level (Schibler and Sassone-Corsi, 2002), the behavioral changes observed here must be a reflection of the alteration of the circadian core transcription/translation feedback loop within the SCN. Indeed, rapid resetting by photic cues was confirmed in the SCN in vivo (Best et al., 1999) and in vitro (Asai et al., 2001). Because mPer2 is essential for the timing of the core feedback loop in the mammalian circadian clock (Hastings et al., 2003), we 

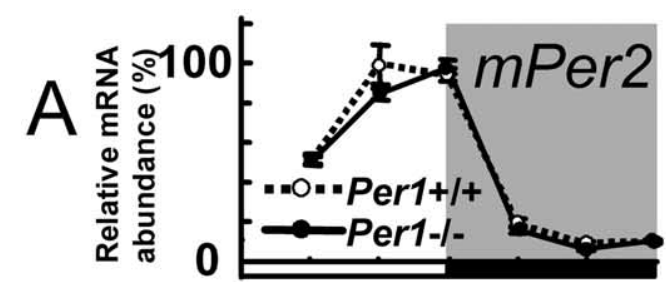

$\begin{array}{llllll}4 & 8 & 121620 & 0\end{array}$

Zeitgeber time (ZT)
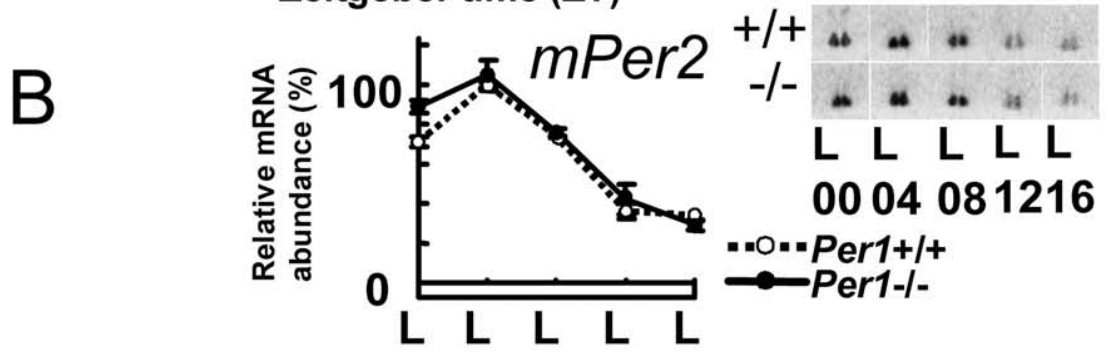

0004081216
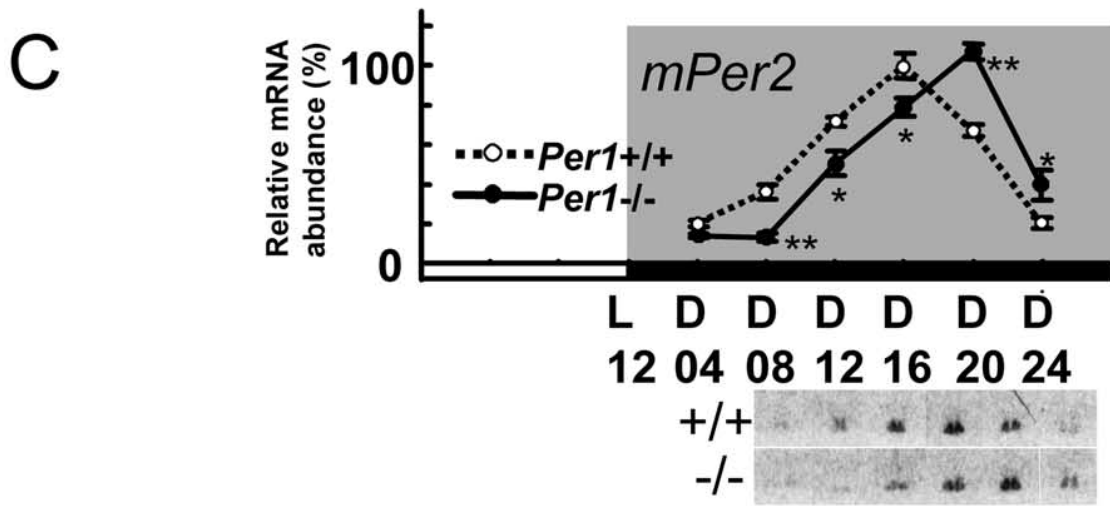

D D D D D D

040812162024
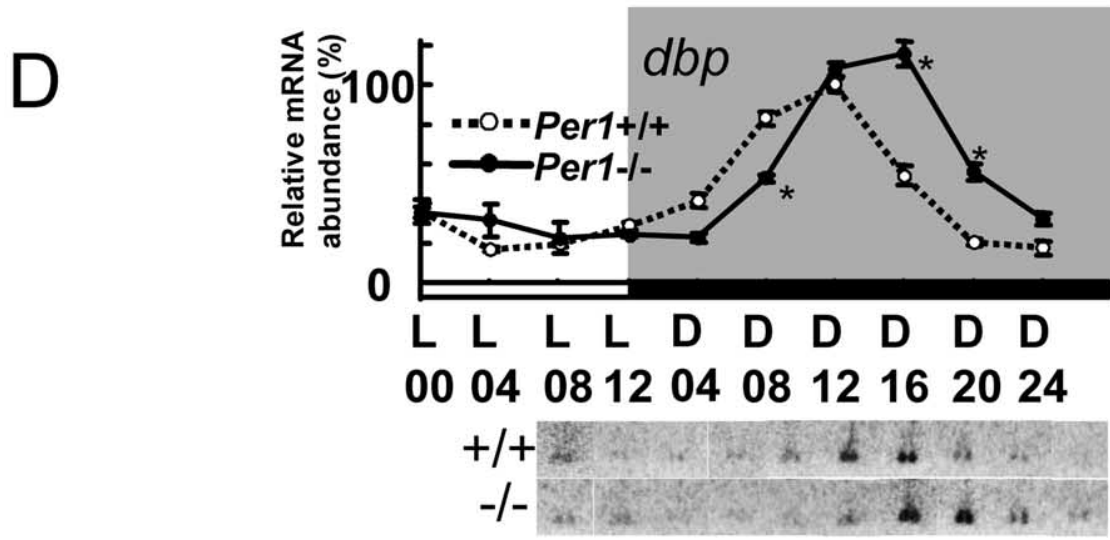

L L L L D D D D D D

00040812040812162024

Figure 2. $\quad A$, Expression profiles of $m P e r 2 m R N A$ in the $S C N$ of $m P e r 1^{+/+}$and $m P e r 1^{-/-}$mice in $L D$. Open circles $\left(m P e r 1^{+/+}\right.$) and filled circles $\left(\mathrm{mPer}^{-1-}\right.$ ) indicate quantified values of $m$ Per2 $\mathrm{mRNA}$ (mean $\left.\pm \mathrm{SEM} ; n=4\right)$ ). $\boldsymbol{B}$, Expression profiles of $m P e r 2$ mRNA in the SCN of $\mathrm{mPer}^{+/+}$and $\mathrm{mPer} 1^{-/-}$mice during the prolongation of light for $16 \mathrm{~h}$. Open circles $\left(\mathrm{mPer}^{+/+}\right)$and filled circles $\left(m P e r 1^{-1-}\right.$ ) indicate quantified values of $m P e r 2 m R N A$ (mean $\pm S E M ; n=4$ ). C, Expression of $m P e r 2 m R N A$ in the $S C N$ of $\mathrm{mPer}^{+/+}$and $\mathrm{mPer}^{-/-}$mice after the $12 \mathrm{~h}$ prolongation of light. Open circles $\left(\mathrm{mPer} 1^{+/+}\right)$and filled circles $\left(\mathrm{mPer} \mathrm{r}^{-1-}\right.$ ) indicate quantified values of $m P$ Per $2 \mathrm{mRNA}$ (mean $\pm \mathrm{SEM} ; n=4$ ). The values indicated by asterisks are statistically significant. ${ }^{* *} p \leq 0.0005 ;{ }^{*} p<0.005 \mathrm{mPer}^{+/+}$versus mPer1 ${ }^{-1-}$ (Bonferroni/Dunn). D, Expression of dbp mRNA in the SCN of mPer $1^{+/+}$and $\mathrm{mPer}^{-1-}$ mice during and after the $12 \mathrm{~h}$ prolongation of light. Open circles $\left(\mathrm{mPer}^{+/+}\right)$and filled circles $\left(\mathrm{mPer}^{-1-}\right)$ indicate quantified values of $d b p$ mRNA (mean $\pm \mathrm{SEM} ; n=3$ ). The values indicated by asterisks are statistically significant. ${ }^{*} p<0.0001$, $\mathrm{mPer}^{+/+}$versus $\mathrm{mPer}^{-1-}$ (Bonferroni/Dunn). A representative autoradiogram of the $\mathrm{SCN}$ is attached to each graph. examined $m P e r 2$ expression profiles in the SCN during the light-prolongation task. In the usual $12 \mathrm{~h} \mathrm{LD}$ regimen (ZT0, ZT4, ZT8, ZT12, ZT16, ZT20), in both $m P e r 1^{-/-}$and $m P e r 1^{+/+}$mice, mPer2 mRNA began to increase before dawn, peaked at ZT12, and then steadily decreased by ZT16-20 (two-way ANOVA; $m \mathrm{Perl}^{-/-}$vs $m P e r 1^{+/+} ; p=0.094$ ) (Fig. $2 A)$. In $16 \mathrm{~h}$ of elongated light exposure (from L00 to L16) from lights off at ZT12 (L00), which induces a larger phase delay in $m P e r 1^{-1-}$ mice, $m P e r 2$ transcript levels increased with $4 \mathrm{~h}$ of light prolongation (L04); thereafter, expression decreased during extended light exposure in both $m P e r 1^{-1-}$ and $m P e r 1^{+/+}$mice in a timedependent manner (one-way ANOVA; $p<0.0001$ ) (Fig. 2B). Because $m$ Per1 ${ }^{-1-}$ and $m \mathrm{Merl}^{+/+}$mice display analogous profiles of mPer2 expression (two-way ANOVA; $p=0.128$ ) (Fig. $2 B$ ), lightinduced transient increase of $m P e r 2$ transcription may occur similarly in these mice. These results indicated that the cause of the enhanced delay of behavioral onset by light prolongation in $m \mathrm{Perl}^{-/-}$mice was not attributable to changes at the $m P e r 2$ transcription level.

Core clock oscillation and its output show a larger phase delay in the second cycle after extended light exposure in mPer $1^{-/-}$mice

We then examined mPer 2 expression profiles after the end of a $12 \mathrm{~h}$ light prolongation (LP12 task) with $4 \mathrm{~h}$ intervals (D04, D08, D12, D16, D20, and D24). A LP12 task (12 $\mathrm{h}$ of light prolongation) was chosen because the magnitude of the behavioral phase shift was largest in both genetic backgrounds (Fig. $1 \mathrm{~B}$ ). $\mathrm{mPer}^{+/+}$mice displayed an increase at D08 and a peak at D16 followed by a decrease (Fig. 2C). The increase of $m P e r 2$ expression in $m P e r 1^{-1-}$ mice began at D12 and peaked at D20. Thus, in contrast to similar expression profiles observed during the long light prolongation in $\mathrm{mPer}^{-1-}$ and $m P e r 1^{+1+}$ mice (Fig. $2 B$ ), mPer 2 mRNA peaks $4 \mathrm{~h}$ later in $m \mathrm{merl}^{-/-}$mice (two-way ANOVA; $p<0.0001$ ) (Fig. $2 C$ ). The magnitude of the behavioral phase shift (4-6 h) did not correlate with the first mPer2 mRNA expression profiles in light but correlated with the phases of mPer 2 expression in the next cycle. As an indicator of clock output levels, we next examined the expression profiles of a clockcontrolled gene, $d b p$, the transcription of which is directly regulated by clock genes (Ripperger et al., 2000; Yamaguchi et al., $2000)$. As shown in Figure $2 D, d b p$ expres- 
sion was equivalent in $m P e r 1^{-/-}$and mPer $^{+/+}$mice during the extended-light task (from L00 to L12; two-way ANOVA; $p=0.2696)$. However, after the task, $d b p$ expression in $\mathrm{mPer}^{-1-}$ mice displayed a 4 h delay when compared with $m P e r 1^{+/+}$ mice (from D04 to D24; two-way ANOVA; $p<0.0001)$. These findings suggest that expression of core clock genes and clock-controlled genes did not reflect the behavioral phase shift seen during extended light exposure without mPerl. But after the task, the expression of these genes does reflect the behavioral rhythms; this occurs after the cessation of the lighting task.

\section{Delayed disappearance of mPER2} protein in the SCN of $\mathrm{mPer}^{-/-}$mice after the extended light exposure

Because there is a dissociation between clock gene expression during the extended-light task and the observed behavioral phase shifts, we examined the expression profile of clock protein levels to know whether there is a dissociation between protein expression patterns and behavioral rhythms. In the usual $12 \mathrm{~h} \mathrm{LD}$ regimen, in both $m P e r 1^{-/-}$and $m P e r 1^{+/+}$ mice, mPER 2 protein began to increase at ZT8, peaked at ZT16, and then steadily decreased by ZT0 (Fig. 3A). We examined the expression of mPER2 protein in the SCN during (L00, L04, L08, and L12) and after light prolongation (D04, D08, and D12) (Fig. 3B, left). At L00 (ZT12), mPER2 protein levels were similarly high in both $m P e r 1^{-1-}$ and $m P e r 1^{+/+}$mice. In mPer $1^{+/+}$mice, mPER2 levels began to decrease at L08 and reached minimal expression at D08, to increase thereafter (D12) (Fig. 3B, left). In contrast, the high expression of mPER2 did not decrease until L08 in $m P e r 1^{-/-}$mice, to reach minimal levels at $\mathrm{L} 12$ ( $4 \mathrm{~h}$ later than $m P e r 1^{+/+}$mice) and then to steadily decrease until D12. From L04 to D08, mPER2 levels are clearly higher in $m P e r 1^{-1-}$ mice than that in $m P e r 1^{+/+}$ mice (two-way ANOVA; $p<0.01$ ). We then examined mPER2 protein expression profiles in the next cycle at $4 \mathrm{~h}$ intervals (D16, D20, D24, D28, D32, and D36). In mPer $1^{+/+}$mice, mPER2 at D16 steadily decreased until D32. In $m P e r 1^{-1-}$ mice, however, mPER2 protein increased until D24 and then decreased thereafter (Fig. $3 B$, right). Clear phase difference $(4-8 \mathrm{~h})$ of mPER2 expressions between $m P e r 1^{-/-}$mice and $m P e r 1^{+/+}$mice was observed in the second cycle (two-way ANOVA; $p<0.0001$ ) (Fig. $3 B$, right). These findings reveal that $m$ Per1 regulates mPER2 protein levels without affecting $m P e r 2$ transcription and that the alteration is likely to induce profound changes within transcription of core clock components and clock-controlled genes for the next cycle, which is then reflected at behavioral level.

mPer1-deficient mice cannot adapt to environmental light/ dark cycles consisting of long complete photoperiods of dim light or long skeleton photoperiods

The larger phase resetting that we observed after several hours of light prolongation raised a possibility that $m P e r 1^{-/-}$mice have a difficulty in entraining to a long-day environment. It is known that expression profiles of $\mathrm{Per}$ genes alter in response to photoperiodic change (Steinlechner et al., 2002). First, we put animals in a long-day schedule: we exposed $m P e r 1^{-/-}$and $m P e r 1^{+/+}$ mice to LD 20/4 h (light on from ZT0 to ZT20; $\mathrm{mPer}^{-1-}, n=14$; $m$ Per $1^{+/+}, n=12 ; 200$ lux illumination) (Fig. 4A-C) and LD $17 / 7 \mathrm{~h}\left(\mathrm{mPer}^{-/-}, n=8 ; m \operatorname{mer} 1^{+/+}, n=8 ; 200\right.$ lux illumination $)$ (Fig. 4D-F). During the period of observation (3-10 weeks), all mice entrained to the both long-day tasks. Because this entrainability is also influenced by the intensity of light, we decrease the intensity of luminescence to $\sim 0.3$ lux, adding the long-day schedules in the first group (LD 20/4 h group). It is possible that low light intensity ( 0.3 lux) (Fig $4 A-C, G$ ) conditions are more natural for nocturnal rodents than the classically high light intensity (200-300 lux) in which usual laboratory animals are housed, because nocturnal rodents live in dim light or darkness in daytime. Nine of $14 \mathrm{mPer}^{-1-}$ mice could not entrain to the dim long-day photoperiod in $35 \mathrm{~d}$ (Fisher's exact probability test; $p<$ 0.005 ) (Fig. $4 G$ ) by phase delaying (Fig. $4 A$ ) and advancing (Fig. $4 B)$. In contrast, all $m P e r 1^{+/+}$mice kept entrained to the long complete photoperiod for $>35 \mathrm{~d}$ (Fig. $4 C, G$ ). However, low light conditions per se are not enough to entrain the rhythm, because all control mice ( $\left.m P e r 1^{-/-}, n=8 ; m P e r 1^{+/+}, n=6\right)$ of the first group kept entrained to LD $12 \mathrm{~h}$ lighting cycle in 0.3 lux for $>35$ $\mathrm{d}$ (data not shown; Fisher's exact probability test; $p=0.65$ ). 

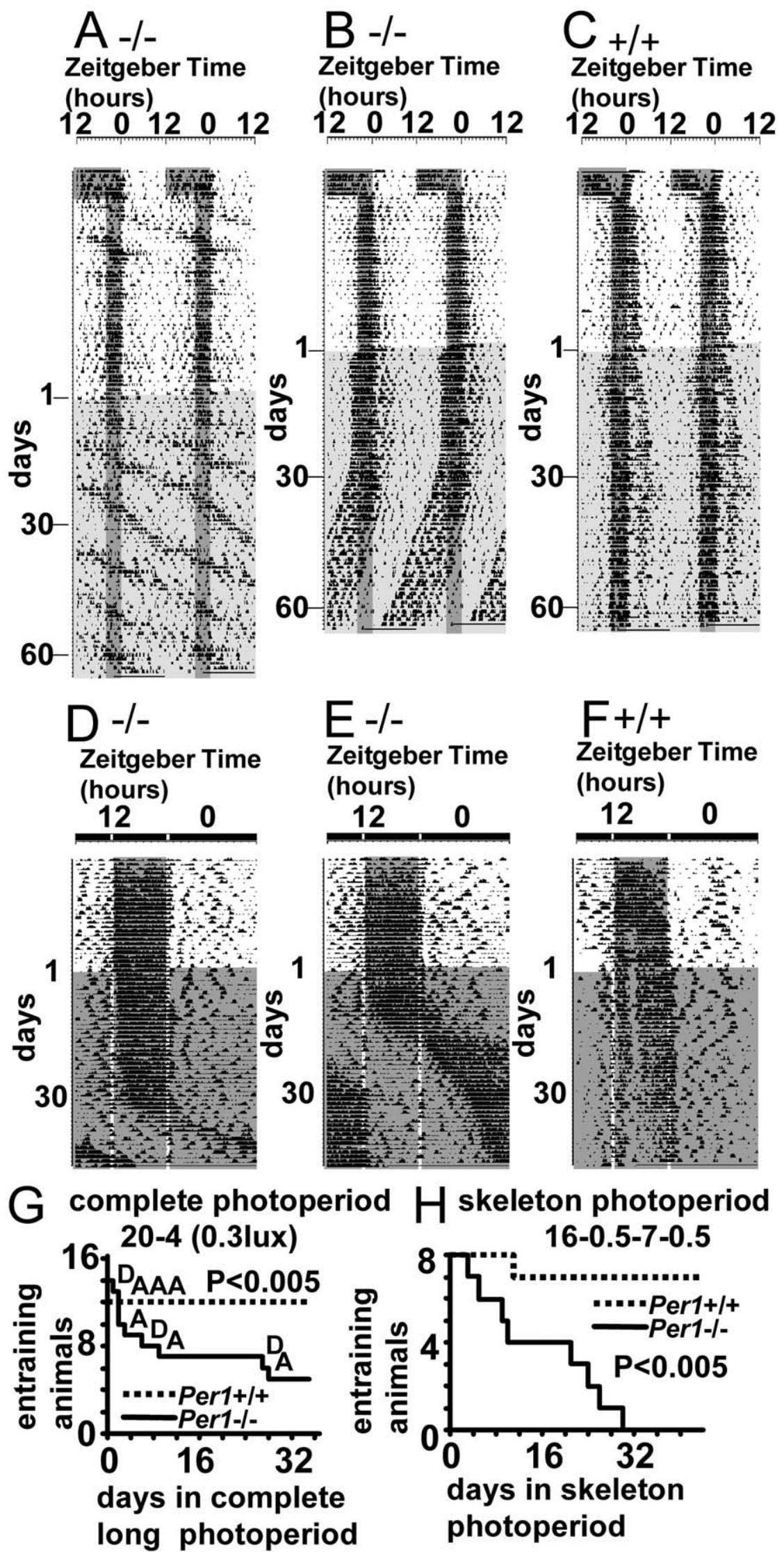

Figure 4. $\quad \boldsymbol{A}-\boldsymbol{C}$, Representative actograms of $\operatorname{Per}^{-1-}(\boldsymbol{A}, \boldsymbol{B})$ and $\operatorname{Per} 1^{+/+}(\boldsymbol{C})$ mice in complete photoperiod with dim light (double plot). Mice were moved (day 1, ZT12) to complete photoperiod with dim light (LD 20/4 h, 0.3 lux) after the same photoperiod with bright light (200 lux). Periods of dim light and complete darkness are indicated by light and dark gray backgrounds, respectively. $\boldsymbol{D}-\boldsymbol{F}$, Representative actograms of $\operatorname{Per}^{-/-}(\boldsymbol{D}, \boldsymbol{E})$ and $\operatorname{Per} 1^{+/+}(\boldsymbol{F})$ mice housed in skeleton photoperiod
Next, a second group (LD 17/7 h group; light on, ZT19; lights off, ZT12) was exposed to skeleton photoperiods (Fig. 4D-F,H). Johnson et al. (2003) speculated that in the case of nocturnal animals, a skeleton photoperiod mimics natural seasonal lighting changes better than complete photoperiods. DeCoursey (1986) suggests that nocturnal rodents in nature reset their clocks by short light exposures. In her simulation experiments, nocturnal rodents (flying squirrels) housed in cages containing dark nest boxes returned to their nest during daytime, and therefore they reset their clocks by short periods (only several minutes per day) of daily light sampling. After entrainment to a $\mathrm{LD} 17 / 7 \mathrm{~h}$ lighting cycle for 3 weeks, mice were moved to skeleton photoperiods [L/D/L/D, 0.5/16/0.5/7 h; light on from ZT19 to ZT19.5 and from ZT11.5 to ZT12 (the beginning of the skeleton photoperiod being ZT19)]. As shown in Figure $4, D$ and $E$, all eight $m P e r 1^{-1-}$ mice could not entrain to the skeleton photoperiod during $30 \mathrm{~d}$. In contrast, as shown in Figure $4 F$, seven of eight $m P e r 1^{+/+}$mice kept entrained to the skeleton photoperiod for $44 \mathrm{~d}$ (Fisher's exact probability test; $p<0.005$ ) (Fig. $4 H$ ). In this experimental condition, all desynchronized mice moved to another light interval by delaying the clock. In contrast, after entrainment to a LD $12 \mathrm{~h}$ lighting cycle for $>3$ weeks, control mice (mPer $1^{-/-}, n=8$; $m$ Per $1^{+/+}, n=8$ ) of the second group were moved to a skeleton photoperiod [L/D/L/D, 0.5/11/0.5/12 h; light on from ZT0 to ZT0.5 and from ZT11.5 to ZT12 (the beginning of skeleton photoperiod being ZT0]. In this skeleton photoperiod, seven of eight $m P e r 1^{-1-}$ mice and all eight $m$ Per $1^{+/+}$mice remained entrained for $44 \mathrm{~d}$ (data not shown; Fisher's exact probability test; $p=0.5)$. Thus, whether synchronization occurs is a result of the difficulty of the task, and we speculate that $m P e r 1^{-1-}$ mice have weaker adaptation ability to environmental light/dark cycle.

(single plot). Mice were moved (day 1, ZT19) to skeleton photoperiod (L/D/L/D, 0.5/16/0.5/7 h) after complete photoperiod (LD 17/7 h). Periods of darkness are indicated by gray backgrounds. G, Time course of the number of entrained animals in complete photoperiod. mPer $1^{-1-}$ mice desynchronized the activity rhythm from light cycle by phase delaying (D) or advancing (A). $\boldsymbol{H}$, Time course of the number of entrained animals in the skeleton photoperiod. The day of desynchronization is determined when the activity onsets began to advance or delay. 


\section{Discussion}

In the present study, we adopted a strong-lighting task to test the role of mPer1 in clock resetting. The prolongation of the lighting period clearly induces a larger-delay phase shift of the behavioral rhythm in $m P e r 1^{-1-}$ mice. Compared with wild-type mice, in $m P e r 1^{-1-}$ mice, long light exposure initially changed mPER2 protein levels, although the expression profiles of mPer 2 at mRNA level was not altered. This result suggests that mPer 1 suppresses synthesis or enhances decay of light induced mPER2 in the SCN. In mPer1 ${ }^{-1-}$ mice, this increased mPER2 may cause larger behavioral phase delay, because mPer 2 mutant show attenuated phase delay by light pulse (Spoelstra et al., 2004). It is possible that desynchronization from the photoperiod (Fig. 4) is caused by the stability of mPER 2 in $m P e r 1^{-1-}$ mice.

These findings raise the attractive possibility that MPER 1 represses light-induced mPER2 at the protein level and attenuates phase resetting by light. Because this process is abolished in mPer1 knock-out mice, environmental light information readily changes the phases of these animals. Although the molecular process involved is unknown at present, the dimerization of mPER1 and mPER2 (Zylka et al., 1998; Field et al., 2000; Yagita et al., 2000) may provide a likely explanation, because it could indeed influence the phosphorylation and/or ubiquitine proteasomedependent mPER2 degradation.

$m P e r 1^{-1-}$ mice could not entrain to experimental long photoperiod with dim light or to skeleton photoperiods. These conditions (dim light, skeleton photoperiod) may mimic the natural lighting schedule for nocturnal rodents. From the present investigation, therefore, it is possible that a nocturnal animal that has $m$ Per 1 has an advantage for entrainment to environmental light/ dark cycles.

One essential issue is whether the duplication of clock genes in mammals has happened only to ensure functional redundancy or whether it has a physiological significance in the context of evolution. Our study underscores the importance of mPer 1 in entrainment to dim long photoperiods and long skeleton photoperiods, conditions that mimic seasonal changes of day/night (long-day condition) for nocturnal rodents living in nature. Our results demonstrate that mPer1 appears to perform a function completely distinct from mPer2, because it is involved in the plasticity of the circadian system, allowing it to adapt to changing photoperiodic cycles.

\section{References}

Albrecht U, Zheng B, Larkin D, Sun ZS, Lee CC (2001) MPer1 and mper2 are essential for normal resetting of the circadian clock. J Biol Rhythms 16:100-104.

Asai M, Yamaguchi S, Isejima H, Jonouchi M, Moriya T, Shibata S, Kobayashi M, Okamura H (2001) Visualization of mPerl transcription in vitro: NMDA induces a rapid phase shift of mPerl gene in cultured SCN. Curr Biol 11:1524-1527.

Bae K, Jin X, Maywood ES, Hastings MH, Reppert SM, Weaver DR (2001) Differential functions of mPer $1, \mathrm{mPer} 2$, and $\mathrm{mPer} 3$ in the $\mathrm{SCN}$ circadian clock. Neuron 30:525-536.

Ban Y, Shigeyoshi Y, Okamura H (1997) Development of vasoactive intestinal peptide mRNA rhythm in the rat suprachiasmatic nucleus. J Neurosci 17:3920-3931.

Best JD, Maywood ES, Smith KL, Hastings MH (1999) Rapid resetting of the mammalian circadian clock. J Neurosci 19:828-835.

Cermakian N, Monaco L, Pando MP, Dierich A, Sassone-Corsi P (2001) Altered behavioral rhythms and clock gene expression in mice with a targeted mutation in the Period1 gene. EMBO J 20:3967-3974.
Daan S, Pittendrigh CS (1976a) A functional analysis of circadian pacemakers in nocturnal rodents II. The variability of phase response curves. J Comp Physiol 106:253-266.

Daan S, Pittendrigh CS (1976b) A functional analysis of circadian pacemakers in nocturnal rodents III. Heavy water and constant light: homeostasis of frequency? J Comp Physiol 106:267-290.

DeCoursey PJ (1986) Light-sampling behavior in photoentrainment of a rodent circadian rhythm. J Comp Physiol [A] 159:161-169.

Dunlap JC (1999) Molecular bases for circadian clocks. Cell 96:271-290.

Field MD, Maywood ES, O’Brien JA, Weaver DR, Reppert SM, Hastings MH (2000) Analysis of clock proteins in mouse SCN demonstrates phylogenetic divergence of the circadian clockwork and resetting mechanisms. Neuron 25:437-447.

Hastings MH, Reddy AB, Maywood ES (2003) A clockwork web: circadian timing in brain and periphery, in health and disease. Nat Rev Neurosci 4:649-661.

Johnson CH, Elliot J, Foster R, Honma K, Kronauer R (2003) Fundamental properties of circadian rhythms. In: Chronobiology: biological timekeeping (Dunlap JC, Loros JJ, DeCoursey PJ, eds), pp 67-105. Sunderland, MA: Sinauer.

Klein DC, Reppert SM, Moore RY (1991) Suprachiasmatic nucleus: the mind's clock. New York: Oxford UP.

Masubuchi S, Honma S, Abe H, Ishizaki K, Namihira M, Ikeda M, Honma K (2000) Clock genes outside the suprachiasmatic nucleus involved in manifestation of locomotor activity rhythm in rats. Eur J Neurosci 12:4206-4214.

Matsuo T, Yamaguchi S, Mitsui S, Emi A, Shimoda F, Okamura H (2003) Control mechanism of the circadian clock for timing of cell division in vivo. Science 302:255-259.

Pando MP, Morse D, Cermakian N, Sassone-Corsi P (2002) Phenotypic rescue of a peripheral clock genetic defect via SCN hierarchical dominance. Cell 110:107-117.

Ripperger JA, Shearman LP, Reppert SM, Schibler U (2000) CLOCK, an essential pacemaker component, controls expression of the circadian transcription factor DBP. Genes Dev 14:679-689.

Schibler U, Sassone-Corsi P (2002) A web of circadian pacemakers. Cell 111:919-922.

Shigeyoshi Y, Taguchi K, Yamamoto S, Takekida S, Yan L, Tei H, Moriya T, Shibata S, Loros JJ, Dunlap JC, Okamura H (1997) Light-induced resetting of a mammalian circadian clock is associated with rapid induction of the mPer1 transcript. Cell 91:1043-1053.

Spoelstra K, Albrecht U, van der Horst GT, Brauer V, Daan S (2004) Phase responses to light pulses in mice lacking functional per or cry genes. J Biol Rhythms 19:518-529.

Steinlechner S, Jacobmeier B, Scherbarth F, Dernbach H, Kruse F, Albrecht U (2002) Robust circadian rhythmicity of Per1 and Per2 mutant mice in constant light, and dynamics of Per1 and Per2 gene expression under long and short photoperiods. J Biol Rhythms 17:202-209.

Takumi T, Matsubara C, Shigeyoshi Y, Taguchi K, Yagita K, Maebayashi Y, Sakakida Y, Okumura K, Takashima N, Okamura H (1998) A mammalian ortholog of Drosophila timeless, highly expressed in SCN and retina, forms a complex with mPER1. Genes Cells 3:167-176.

Yagita K, Yamaguchi S, Tamanini F, van Der Horst GT, Hoeijmakers JH, Yasui A, Loros JJ, Dunlap JC, Okamura H (2000) Dimerization and nuclear entry of mPER proteins in mammalian cells. Genes Dev 14:1353-1363.

Yamaguchi S, Mitsui S, Yan L, Yagita K, Miyake S, Okamura H (2000) Role of DBP in the circadian oscillatory mechanism. Mol Cell Biol 20:4773-4781.

Zheng B, Larkin DW, Albrecht U, Sun ZS, Sage M, Eichele G, Lee CC, Bradley A (1999) The mPer2 gene encodes a functional component of the mammalian circadian clock. Nature 400:169-173.

Zheng B, Albrecht U, Kaasik K, Sage M, Lu W, Vaishnav S, Li Q, Sun ZS, Eichele G, Bradley A, Lee CC (2001) Nonredundant roles of the mPer1 and mPer2 genes in the mammalian circadian clock. Cell 105:683-694.

Zylka MJ, Shearman LP, Levine JD, Jin X, Weaver DR, Reppert SM (1998) Molecular analysis of mammalian timeless. Neuron 21:1115-1122. 